\title{
Characterization by electrospray ionization and tandem mass spectrometry of rhamnolipids produced by two Pseudomonas aeruginosa strains isolated from Brazilian crude oil
}

\author{
Jorge F.B. Pereira, ${ }^{a}$ Eduardo J. Gudiña, ${ }^{b}$ M. Luísa Dória, ${ }^{c}$ M. Rosário Domingues, ${ }^{c}$ Lígia R. Rodrigues, ${ }^{b}$ \\ José A. Teixeirab and João A.P. Coutinho ${ }^{a}$ \\ ${ }^{a}$ CICECO-Chemistry Department, University of Aveiro, Portugal. E-mail: jcoutinho@ua.pt \\ blBB-Institute for Biotechnology and Bioengineering, Centre of Biological Engineering, University of Minho, Braga, Portugal \\ 'Mass Spectrometry Center, Department of Chemistry, University of Aveiro, Portugal
}

\begin{abstract}
In this work, biosurfactants produced by two Pseudomonas aeruginosa strains isolated from Brazilian crude oils were identified by proton nuclear magnetic resonance ('H NMR) and further characterized by mass spectrometry (MS) coupled with electrospray ionization (ESI) and tandem mass spectrometry (MS/MS) analysis in positive mode and their surface activities evaluated. Mono-rhamnolipids and di-rhamnolipids were identified for both isolates, but the most abundant were found to be mono-rhamnolipids. The similarity of rhamnolipids produced by the two strains was in good agreement with their surface activities. Both biosurfactants exhibited similar aqueous solution surface tensions, high emulsification indexes and critical micelle concentration values. The results obtained show that ESI-MS and MS/MS analysis alone provide a fast and highly specific characterization of biosurfactants produced by microbial strains.
\end{abstract}

Keywords: rhamnolipids, Pseudomonas aeruginosa, biosurfactants, mass spectrometry, ESI-MS

\section{Introduction}

Surface-active agents or surfactants are amphiphilic molecules that comprise both hydrophobic and hydrophilic moieties, which are able to reduce surface and inter facial tensions and can form and stabilize oil in water or water in oil emulsions. ${ }^{1}$ These compounds have several applications and traditionally are used in food, pharmaceutical, cosmetics and petroleum industries. ${ }^{2}$ Chemical surfactants are widely used in industry but the increase in environmental issues and restrictive laws led to the development of biodegradable and naturally produced surfactants, such as oleo chemical- or sugarbased and microbial surfactants. ${ }^{3}$ Biosurfactants appear as a reliable alternative due to their lower toxicity, higher biodegradability and effectiveness at extreme temperature, salinity and $\mathrm{pH}$ conditions. . $^{5}$

Several biosurfactants have been reported, but the most important groups include glycolipids and lipopeptides. ${ }^{2}$ Glycolipids consist of a carbohydrate moiety linked to longchain aliphatic acids or hydroxyl aliphatic acids. ${ }^{6}$ Among them, the most relevant are the rhamnolipids produced by the Pseudomonas species due to their good surface activity and emulsifying properties. ${ }^{7,8}$ Rhamnolipids are composed of one or two $\beta$-hydroxy fatty acids of different chain lengths (C8-C22) linked to a mono-rhamnose (Rha-) or di-rhamnose (RhaRha) unit. 6,9 The two major rhamnolipid groups are the monorhamnolipids and di-rhamnolipids; however, there are more 
than 58 rhamnolipid congeners and homologs with different combinations of saturated and unsaturated acyl chains and sugar moieties. ${ }^{2,7,8,10,11}$

Mass spectrometry (MS) with electrospray ionization (ESI) is a powerful analytical technique that has been commonly used for the identification and analysis of several biomolecules, such as lipids ${ }^{12}$ and carbohydrates. ${ }^{13}$ Using this approach, it is possible to obtain information about the structural details of the analyzed molecules. Moreover, taking into account that rhamnolipids contain both lipids and carbohydrates in their structure, these molecules are ideal to be analyzed by MS with ESI. This technique has the advantage of allowing the analysis of very small amounts of sample, even when present in mixtures and without derivatization. However, information on the analysis of rhamnolipids by MS is scarce; thus, this study constitutes an important approach to fill this necessity. Until now, the characterization of the structure of rhamnolipids using MS focused mainly on the use of ESI-MS analysis in negative mode ${ }^{10,14-18}$ and only one study reported the use of MS in positive mode, using matrix-assisted laser desorption/ionization (MALDI). ${ }^{19}$ Among these studies, only the work carried out in the negative mode suggested the use of tandem MS (MS/MS) for the identification of the structure details of these biomolecules. ${ }^{14}$ Even if these works performed the study of the fragmentation by increasing the cone voltage ion of the ESI source, after MS had been coupled with liquid chromatography (LC), this is not a truly MS/MS analysis. In addition, analysis of the fragmentation pathways of rhamnolipid positive ions was not previously performed or reported. In positive mode the rhamnolipid ionized preferentially as $[\mathrm{M}+\mathrm{Na}]^{+}$ ions, as identified by Price and collaborators. ${ }^{19}$ However, these authors did not confirm the structural features of the rhamnolipids identified by MS/MS. In fact, MS spectra with the identification of the $[\mathrm{M}+\mathrm{Na}]^{+}$ions allowed the identification of the molecular weight of compounds, while the identification and confirmation of the structural features can only be achieved using MS/MS strategies.

Therefore, in this work, the chemical characterization of rhamnolipids produced by two different strains of Pseudomonas aeruginosa will be conducted using the ${ }^{1} \mathrm{H}$ NMR and MS approach based on ESI-MS and MS/MS techniques in positive mode. Furthermore, a detailed analysis of the fragmentation pathways observed in the MS/MS spectra of the $[\mathrm{M}+\mathrm{Na}]^{+}$ions will be performed to confirm the structural features of the glycolipids. The surface-active and emulsification properties of rhamnolipid solutions will be evaluated, as well as the relationship between these features and the chemical structure of the rhamnolipids.

\section{Experimental}

\section{Isolation of microorganisms from crude oil samples and biosurfactant production and recovery}

Crude oil samples from four wells at a Brazilian oil field were collected in sterile bottles. For the isolation of microorganisms, enrichment cultures were performed as described previously. ${ }^{20}$ Biosurfactant production by the selected strains was carried out in shake flasks containing $500 \mathrm{~mL}$ of LB medium. Each flask was inoculated with $5 \mathrm{~mL}$ of a pre-culture that was prepared using the same culture medium and incubated overnight at $40^{\circ} \mathrm{C}$ and $120 \mathrm{rpm}$. The flasks were then incubated under the same conditions until the stationary phase was reached. At the end of the fermentation, the cells were harvested by centrifugation $(10000 \times \mathrm{g}$, $20 \mathrm{~min}, 10^{\circ} \mathrm{C}$ ) and cell dry weight ( $\left.\mathrm{g} \mathrm{L}^{-1}\right)$ was determined. To recover the biosurfactant, the cell-free supernatant was subjected to acid precipitation. After the precipitation, the crude biosurfactant was dissolved in a minimal amount of demineralised water and the $\mathrm{pH}$ was adjusted to 7.0 using $1 \mathrm{M}$ $\mathrm{NaOH}$. Finally, the crude biosurfactant solution was freezedried and the product obtained was weighed and stored at $-20^{\circ} \mathrm{C}$ for further use.

\section{Identification of the isolates}

Bacterial isolates that displayed high biosurfactant production were selected and identified by partial 165 rRNA sequencing. The $16 \mathrm{~S}$ rRNA gene was amplified by PCR using primers $341 \mathrm{~F}$ and $907 \mathrm{R}$. The resulting sequences were compared with sequences in the GenBank database of the National Center for Biotechnology Information (NCBI) (http:// www.ncbi.n(m.nih.gov) using the nucleotide-nucleotide blast (BLASTn) network service, to determine their phylogenetic affiliations.

\section{Extraction and purification of rhamnolipids}

Rhamnolipids were extracted from cell-free supernatants, using the Folch extraction commonly used to extract lipids from biomolecules. The Folch extraction procedure was performed as described elsewhere, ${ }^{21}$ briefly, by adding $2: 1$ chloroform/methanol mixture to the supernatant sample to achieve a final ratio of chloroform/methanol/water (8:4:3). The mixture was centrifuged $(9000 \times \mathrm{g}, 5 \mathrm{~min})$, the organic layer was collected and the samples were evaporated to dryness under $\mathrm{N}_{2}$ at $37^{\circ} \mathrm{C}$ for $30 \mathrm{~min}$. Prior to MS and NMR analysis, the samples were re-dissolved in chloroform.

\section{Mass spectrometry}

Analysis of rhamnolipids was carried out by MS using ESI ionization obtained in a Q-ToF 2 (Micromass, Manchester, UK). ESI conditions in the electrospray Q-Tof mass spectrometer were as follows: electrospray voltage was $3 \mathrm{kV}$ in the positive mode with a cone voltage of $30 \mathrm{~V}$. The source temperature was $80^{\circ} \mathrm{C}$ and the desolvation temperature was $150^{\circ} \mathrm{C}$. MS/ MS spectra were performed using argon as collision gas and energy between 30-40V. Data was acquired using a Mass Lynx data system (V4.0).

\section{NMR spectroscopy}

The purified rhamnolipids were re-dissolved in deuterated chloroform $\left(\mathrm{CDCl}_{3}\right)$ and the respective ${ }^{1} \mathrm{H}$ NMR spectra were recorded using a BrukerAvance 300 spectrometer operating at $300.13 \mathrm{MHz}$. 


\section{Results and discussion Isolation and identification of biosurfactant- producing microorganisms}

Isolates \#111 and \#902 were identified according to the partial sequence obtained from their $16 \mathrm{~S}$ rRNA genes. The sequences obtained were compared with those described in the databases and both showed $100 \%$ similarity with $P$. aeruginosa. The sequences were deposited in the GenBank database under accession numbers JQ281109 and JQ281110. The sequences obtained for both isolates showed $99 \%$ similarity. This fact, together with the different morphology exhibited by both isolates growing in solid and liquid media, confirms that they are different isolates.

\section{NMR spectroscopy analysis}

The composition of the biosurfactants obtained from isolates \#111 and \#902 was probed by ${ }^{1} \mathrm{H}$ NMR analysis. The respective ${ }^{1} \mathrm{H}$ chemical shifts are shown in Table 1 and respective spectra presented in Supplementary Data.

The ${ }^{1} \mathrm{H}$ NMR spectra were in good agreement with those obtained by other authors ${ }^{6,10,19}$ showing that the purified surfactant samples presented the chemical shift deviations characteristic of rhamnolipid homologs. The spectrum of isolate \#902 showed signals related to a sugar moiety at $\delta$ $4.82\left(C-1^{\prime}\right), 3.63-3.78\left(C-2^{\prime}, C-3^{\prime}, C-5^{\prime}\right), 3.48\left(C-4^{\prime}\right)$ and 1.24 $\left(\mathrm{C}-\mathrm{6}^{\prime}\right)$ related to a rhamnose moiety. A similar spectrum was also obtained for isolate \#111 at, respectively, 84.86 (C-1'), 3.62-3.80 (C-2', C-3', C-5'), $3.47\left(C-4^{\prime}\right)$ and $1.24\left(C-6^{\prime}\right)$. In addition, both spectra showed the hydroxy fatty acid signals. As ${ }^{1} \mathrm{H}$ NMR was used, it was not possible to define the rhamnolipids structure precisely, thus these were further characterized by ESI-MS and MS/MS as described below.

\section{Mass spectrometry}

The ESI-MS spectra, obtained in positive mode, of the extracts obtained for each isolate (\#111 and \#902) are shown in Figure 1. In both ESI-MS spectra, it is possible to identify several sodium adducts, $[\mathrm{M}+\mathrm{Na}]^{+}$ions, from the rhamnolipids present in the samples. The corresponding potassium adducts, $[\mathrm{M}+\mathrm{K}]^{+}$ ions, are also observed, thus confirming that rhamnolipid species are present. The formation of these adducts is typical of carbohydrate derivatives ${ }^{13}$ by ESI-MS and was also observed by other researchers during MALDI-MS analysis of rhamnolipids. ${ }^{19}$

The ions identified in the ESI-MS spectra (Figure 1) were attributed to both mono- and di-rhamnolipids with distinct fatty acyl chains, as shown in Table 2 .

The most abundant ions observed in the extracts are monorhamnolipids, namely Rha-C10:0/C10:0, corresponding to $[\mathrm{M}+\mathrm{Na}]^{+}$ion at $\mathrm{m} / \mathrm{z}$ 527.2, followed by Rha-C10:0/C12:0 and Rha-C10:0/C12:1, corresponding to $[\mathrm{M}+\mathrm{Na}]^{+}$ions at $\mathrm{m} / \mathrm{z} 555.3$ and $\mathrm{m} / \mathrm{z}$ 553.3, respectively. The di-rhamnolipids found are Rha-Rha-C10:0/C10:0 and Rha-Rha-C10:0/C12:0, corresponding to $[\mathrm{M}+\mathrm{Na}]^{+}$ions at $\mathrm{m} / \mathrm{z} 673.3$ and $\mathrm{m} / \mathrm{z} 701.3$, respectively (Table 1).

Few differences were found between the two samples analyzed. Although ESI-MS is a qualitative and not a quantitative method, it was possible to notice some differences in the relative abundance of di-rhamnolipid ions comparing samples from \#902 and \#111 isolates. Biosurfactant from isolate \#111 seems to have a minor relative abundance of the Rha-Rha-C10:0/C10:0 and Rha-Rha-C10:0/C12:0 compared to \#902. Although obtained from two $P$. aeruginosa strains, apparently these two samples have a similar composition of rhamnolipids, which could be explained by their similar

Table 1. ${ }^{1} \mathrm{H}$ NMR spectroscopy data from rhamnolipids ( $\delta$ values, $\mathrm{ppm}$ ) produced by two different strains of Pseudomonas aeruginosa (\#111 and \#902).

\begin{tabular}{|c|c|c|c|}
\hline & & \#902 & $\# 111$ \\
\hline Moiety & Proton location & $\delta$ (ppm) & $\delta(p p m)$ \\
\hline \multirow[t]{6}{*}{ Rhamnose(s) } & $\mathrm{C}-1^{\prime}$ & 4.82 & 4.86 \\
\hline & $\mathrm{C}-2^{\prime}$ & $3.63-3.78$ & $3.62-3.80$ \\
\hline & $C-3^{\prime}$ & $3.63-3.78$ & $3.62-3.80$ \\
\hline & $C-4^{\prime}$ & 3.48 & 3.47 \\
\hline & $C-5^{\prime}$ & $3.63-3.78$ & $3.62-3.80$ \\
\hline & $C-6^{\prime}$ & 1.24 & 1.24 \\
\hline \multirow[t]{8}{*}{ Hydroxy fatty acid } & $C-1$ & $4.20-4.22$ & $4.18-4.25$ \\
\hline & $C-2$ & $2.29-2.61$ & $2.15-2.72$ \\
\hline & $C-3$ & - & $1.65-1.69$ \\
\hline & $\mathrm{COOH}$ & - & \\
\hline & $\mathrm{CH}_{3}$ & 0.86 & 0.84 \\
\hline & $C-4$ & $5.28-5.38$ & $5.28-5.46$ \\
\hline & $C-5$ & $2.29-2.61$ & $2.15-2.72$ \\
\hline & $\mathrm{CH}_{2}$ & $1.24-1.58$ & $1.24-1.50$ \\
\hline
\end{tabular}




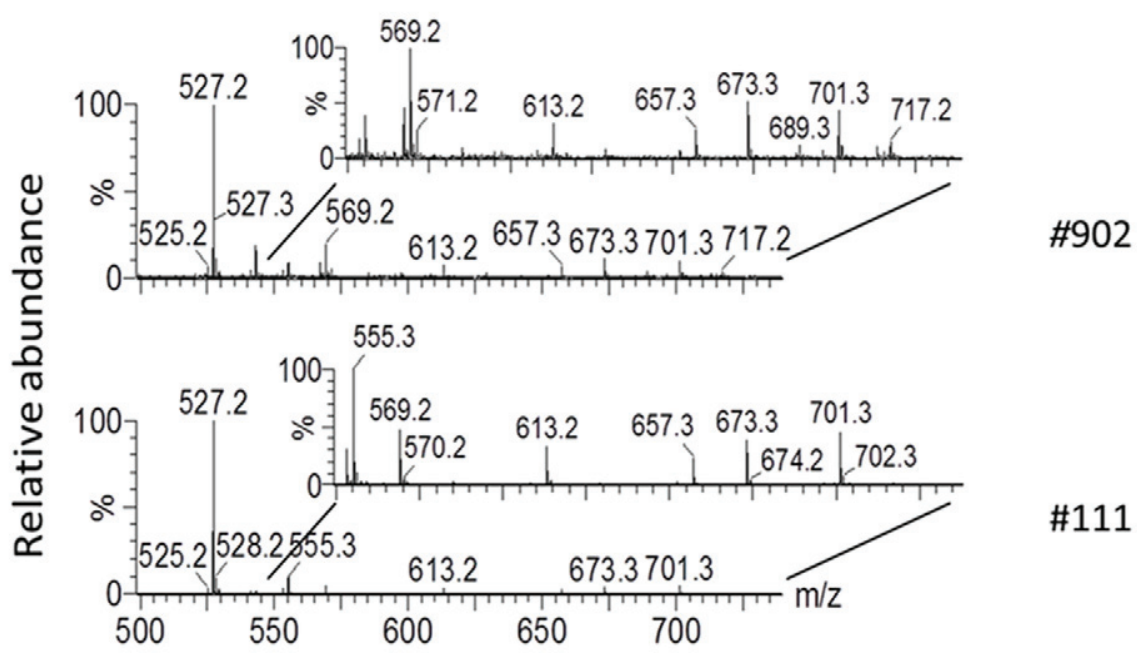

Figure 1. ESI-MS spectra obtained using an electrospray Q TOF mass spectrometer in positive mode of \#111 and \#902 biosurfactants. Y-axis: Relative abundance considering the highest abundant ion as $100 \%$; X-axis: $\mathrm{m} / \mathrm{z}$ for each ion. ESI-MS spectra were acquired in the positive mode.

surface activities and emulsifying properties previously described. ${ }^{20}$ The large CMC values obtained for these biosurfactants ${ }^{20}$ when compared with other rhamnolipids previously reported may be due to two factors: on the one hand, Mata-Sandoval et al. ${ }^{22}$ showed that rhamnolipids with shortchain fatty acids have smaller hydrophobicity and, accordingly, start to form micelles at higher biosurfactant concentrations; on the other hand, the presence of di-rhamnolipids in the mixtures of surfactants obtained from both isolates (\#111 and \#902) also leads to an increase in the CMC values. This behavior has also been observed by Haba et al. ${ }^{23}$ which showed that higher CMC values are obtained due to the contribution of the di-rhamnolipid.

In order to confirm the proposed structure of each species identified by ESI-MS spectra, namely the presence of rhamnose units and the identification of the fatty acyl chain composition, the ESI-MS/MS spectra of each $[\mathrm{M}+\mathrm{Na}]^{+}$ion were conducted and analyzed. This approach allows the identification of the fatty acyl chains length, their position in the rhamnolipid structure and the confirmation of presence of the rhamnose sugar units. Below, a detailed explanation on the typical fragmentation of a mono-rhamnolipid and a di-rhamnolipid is given. Rha-C10:0/C12:0 corresponding to an $[\mathrm{M}+\mathrm{Na}]^{+}$ion at $\mathrm{m} / \mathrm{z} 555.3$ and Rha-Rha-C10:0/C10:0 corresponding to an $[\mathrm{M}+\mathrm{Na}]^{+}$ion at $\mathrm{m} / \mathrm{z} 673.3$ are used as examples.

An MS/MS spectrum of the $[\mathrm{M}+\mathrm{Na}]^{+}$ion of the Rha-C10:0/ C12:0 [Figure 2(a)] shows a product ion at $\mathrm{m} / \mathrm{z} 409$, formed due to the loss of the rhamnose residue $(-146 \mathrm{Da})$ and ions attributed to the rhamnose residue $\left[\mathrm{Rhamnose}_{\text {res }}+\mathrm{Na}\right]^{+}$at $\mathrm{m} / \mathrm{z} 169$ and to rhamnose unit [Rhamnose $+\mathrm{Na}]^{+}$at $\mathrm{m} / \mathrm{z} 187$ as illustrated in Figure 2. Other product ions are observed at $\mathrm{m} / \mathrm{z} 357$ due to the loss of the terminal fatty acyl chain $(-170 \mathrm{Da})$ and also the ions corresponding to the [hydroxy fatty acid $+\mathrm{Na}]^{+}$at $\mathrm{m} / z 239$, identified as the terminal fatty acid $\left(\mathrm{C}_{12} \mathrm{C}_{24} \mathrm{O}_{3}\right)$ and at $\mathrm{m} / \mathrm{z} 211$, identified as the middle fatty acid $\left(\mathrm{C}_{10} \mathrm{H}_{20} \mathrm{O}_{3}\right)$. These fragmentation pathways allow identifying the molecular weight and structure of the fatty acids that constitute the mono-rhamnolipid and their position in the initial molecule.

Moreover, the ESI-MS/MS spectrum of the $[\mathrm{M}+\mathrm{Na}]^{+}$ion of the di-rhamnolipid, Rha-Rha-C10:0/C10:0 [Figure 3(a)], showed product ions formed by the loss of one (-146 Da) and two

Table 2. Identification of $[\mathrm{M}+\mathrm{Na}]^{+}$and $[\mathrm{M}+\mathrm{K}]^{+}$ions observed in the ESI-MS spectra corresponding to mono and di-rhamnolipids present in the total sample (extract).

\begin{tabular}{|c|c|c|c|}
\hline & $m / z[M+K]^{+}$ & $m / z[\mathrm{M}+\mathrm{Na}]^{+}$ & Fatty acyl chains ${ }^{*}$ \\
\hline \multirow[t]{3}{*}{ Mono-rhamnolipids } & 543.2 & 527.2 & C10:0/C10:0 \\
\hline & 569.2 & 553.3 & C10:0/C12:1 \\
\hline & 571.2 & 555.3 & C10:0/C12:0 \\
\hline \multirow[t]{2}{*}{ Di-rhamnolipids } & 689.3 & 673.3 & C10:0/C10:0 \\
\hline & 717.2 & 701.3 & C10:0/C12:0 \\
\hline
\end{tabular}

*The attribution of the fatty acyl chains composition of each molecular species was done accordingly with the interpretation of the correspondent MS/MS spectra; fatty acyls (Cx:y): the first value indicates $x$ number of carbons in the fatty acid chain and the second value $y$ represent the number of double bonds in that chain. 
a)

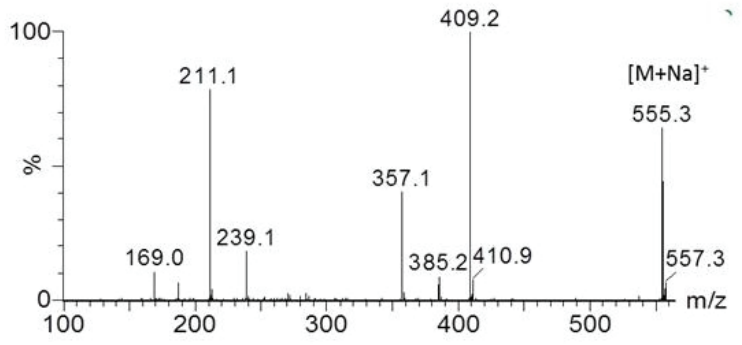

b)

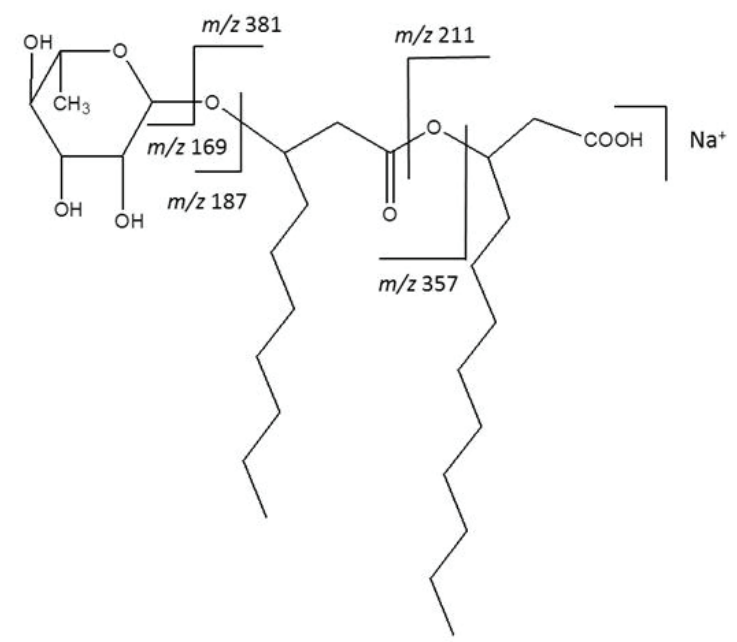

c)
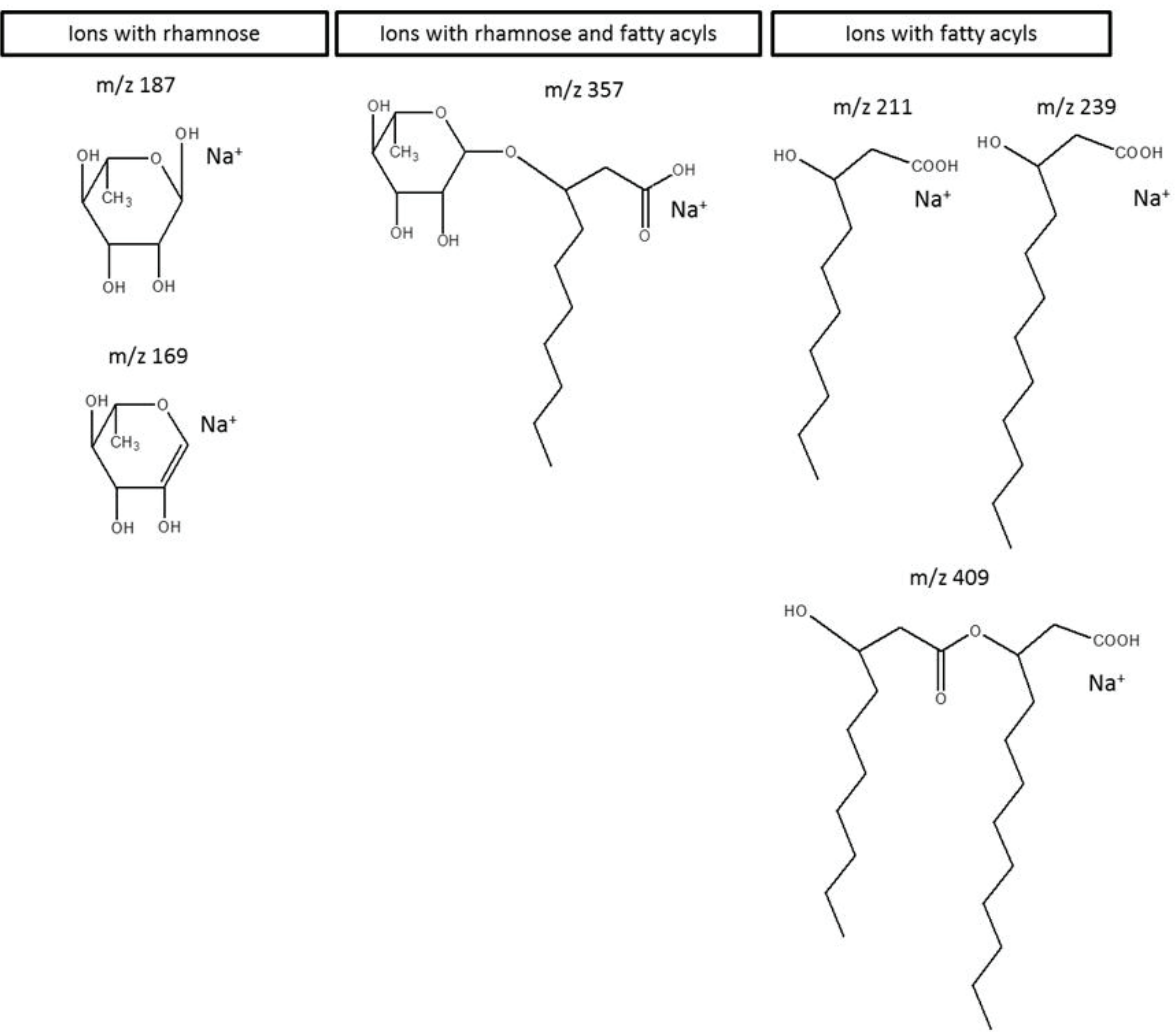

Figure 2. (a) ESI-MS/MS spectrum of the $[M+N a]^{+}$ion at $m / z 555.3$ corresponding to mono-rhamnolipid Rha-C10:0/C12:0 obtained in ESI-QTOF. (b) Molecular structure of Rha-C10:0/C12:0 with the main cleavages correspondent to the fragmentation observed in the $\mathrm{MS} / \mathrm{MS}$ spectrum of $[\mathrm{M}+\mathrm{Na}]^{+}$ion. (c) Molecular structure of the ions originated from the fragmentation of $[\mathrm{M}+\mathrm{Na}]^{+}$ion. 
a)

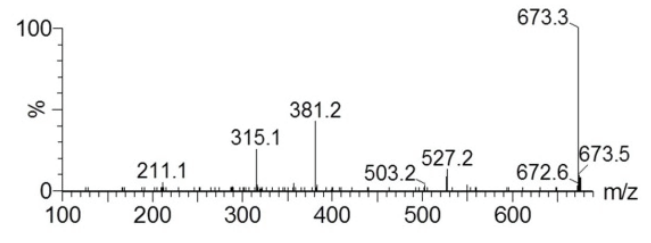

b)

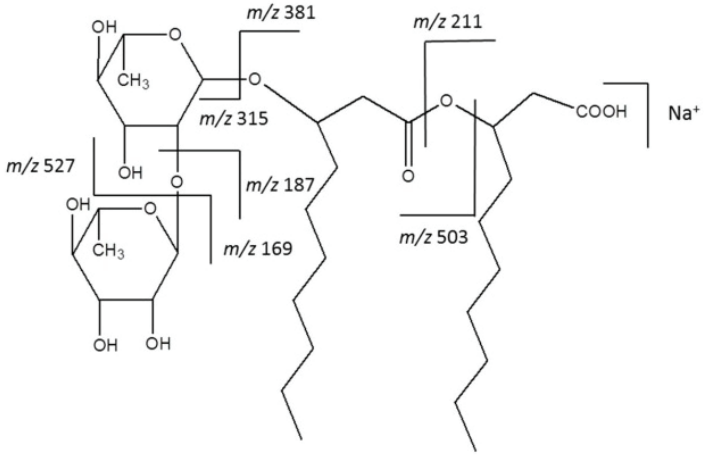

c)

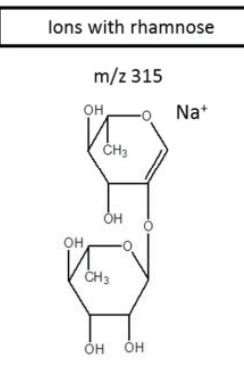

$\mathrm{m} / \mathrm{z} 187$

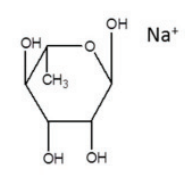

$\mathrm{m} / \mathrm{z} 169$

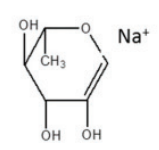

Ions with rhamnose and fatty acyls

lons with fatty acyls
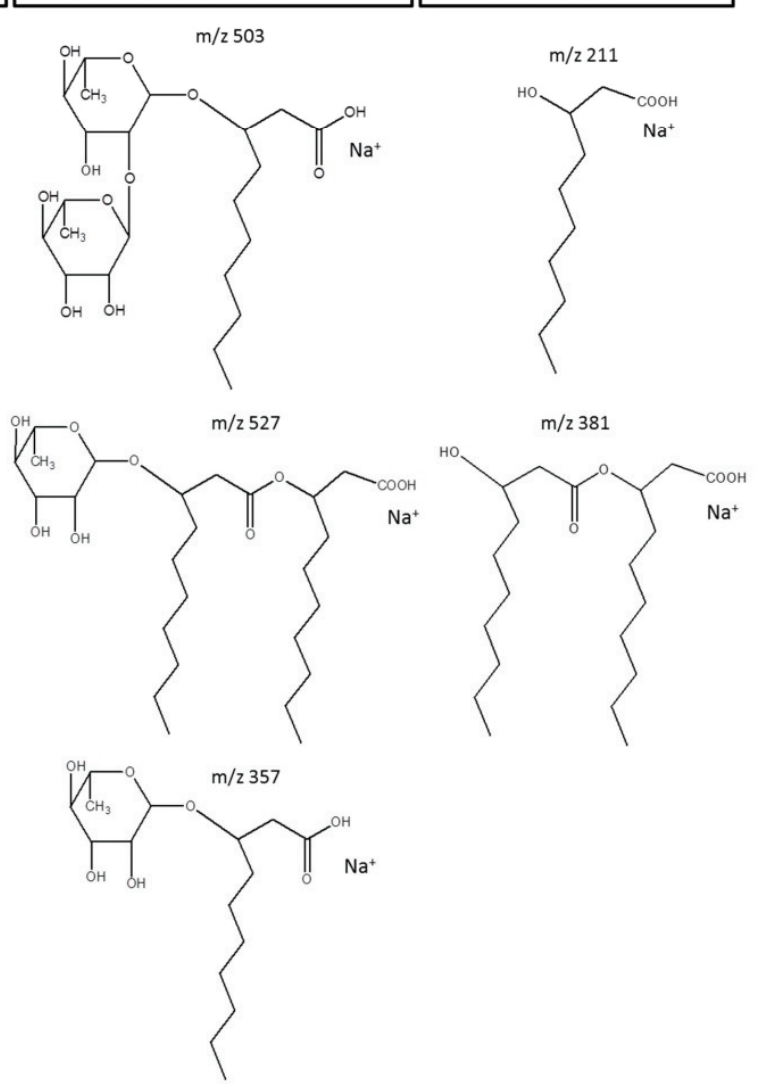

$\mathrm{m} / \mathrm{z} 211$

Hо $\mathrm{COOH}$

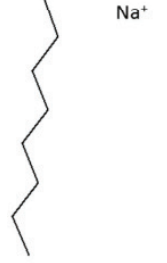

$\mathrm{m} / \mathrm{z} 381$

Figure 3. (a) ESI-MS/MS spectrum of the $[\mathrm{M}+\mathrm{Na}]^{+}$ion at $\mathrm{m} / \mathrm{z} 673.3$ corresponding to di-rhamnolipid Rha-Rha-10:0/10:0 obtained in ESI-Q ToF. (b) Molecular structure of Rha-Rha-10:0/10:0 with the main cleavages correspondent to the fragmentation observed in the $\mathrm{MS} / \mathrm{MS}$ spectrum of $[\mathrm{M}+\mathrm{Na}]^{+}$ion at $\mathrm{m} / \mathrm{z}$ 673.3. (c) Molecular structure of the ions originated from the fragmentation of [M+Na $]^{+}$ion at $\mathrm{m} / \mathrm{z}$ 673.3.

rhamnose residues ( $2 x-146$ Da) with the subsequent formation of the ions at $\mathrm{m} / \mathrm{z} 527$ and $\mathrm{m} / \mathrm{z} 381$, respectively. The product ion at $\mathrm{m} / \mathrm{z} 315$, identified as $\left[\text { Rhamnose }{ }_{2}+\mathrm{Na}\right]^{+}$confirms that both rhamnose units are linked together. Ions attributed to one rhamnose were identified at $\mathrm{m} / \mathrm{z} 169$ [Rhamnose $\left._{\text {res }}+\mathrm{Na}\right]^{+}$ and $\mathrm{m} / z 187$ [Rhamnose $+\mathrm{Na}]^{+}$. In this spectrum, it is possible 
to see other informative ions formed by the loss of the terminal of the fatty acyl chain $\left(-170 \mathrm{Da}--\mathrm{C}_{10} \mathrm{H}_{20} \mathrm{O}_{3}\right)$ at $\mathrm{m} / z 503$, the loss of a fatty acyl and a rhamnose (-316 Da) at $\mathrm{m} / \mathrm{z} 357$ and also the ions corresponding to the fatty acyl chains at $\mathrm{m} / \mathrm{z} 211$.

This approach was used to elucidate the structure of all the rhamnolipids identified and the results are reported in Table 1. There are a few studies that characterize the fragmentation of rhamnolipids with MS/MS, the best known being the rupture of the ester link between two alkyl chains of rhamnolipids and near sugar units in negative mode $\left(\left[\mathrm{M}-\mathrm{H}^{-}\right]^{-}{ }^{6,24}\right.$ In spite of this work having been carried out in positive mode, with the formation of $[\mathrm{M}+\mathrm{Na}]^{+}$, the fragmentations observed are similar, suggesting that they are characteristic of these molecules.

This is one of the first studies on the characterization of rhamnolipids by MS in positive mode, with the formation of $[\mathrm{M}+\mathrm{Na}]^{+}$ions ${ }^{19,25}$ and the first using electrospray ionization. The results reported here are in good agreement with Price et al., ${ }^{19}$ which identified the rhamnolipids' structure with MS in positive mode and MALDI ionization, observing the mono-rhamnolipids Rha-C10:0/C10:0, Rha-C10:0/C12:0 and Rha-C10:0/ C12:1 and the di-rhamnolipids Rha-Rha-C10:0/C10:0 and Rha-Rha-C10:0/C12:0 with the formation of $[\mathrm{M}+\mathrm{Na}]^{+}$ions and a minor part with the formation of $[\mathrm{M}+\mathrm{K}]^{+}$ions. The results obtained herein show that ESI-MS and MS/MS analysis are powerful techniques for characterizing biosurfactants produced by microbial strains, allowing the identification and confirmation of the structural features using only MS/MS strategies, without previous LC analysis.

\section{Conclusion}

In this work, biosurfactants produced by two isolated $P$. aeruginosa strains were characterized. Their structures have been identified by ${ }^{1} \mathrm{H}-\mathrm{NMR}$ as being rhamnolipids and further characterized by ESI/MS and MS/MS as mixtures of mono-rhamnolipids and di-rhamnolipids. The most abundant homologs found in the extracts were the mono-rhamnolipids, Rha-C10:0/C12:0 and Rha-C10:0/ C12:1 and the di-rhamnolipids Rha-Rha-C10:0/C10:0 and Rha-Rha-C10:0/C12:0. The rhamnolipid structural characterization showed that although these surfactants have been obtained from two different $P$. aeruginosa strains, they have a close composition that explains their similar behavior. The structural characterization of the biosurfactants produced by microorganisms is very important to understand their surface-active properties and formation of molecular aggregates. The development of rapid analysis, such as ESI/MS and MS/MS, which allow the characterization with high sensitivity and specificity of the surfactants present in bacterial extracts, is crucial. In conclusion, it is important to understand biosurfactant specificities in order to increase their potential applications as, for example, in bioremediation of environments contaminated with crude oil and in tertiary recovery of crude oil from reservoirs by microbial-enhanced oil recovery processes. In this context, the results herein reported show that ESI-MS and MS/MS analysis can be powerful techniques for a simple and fast structural characterization of biosurfactants produced by microbial strains.

\section{Acknowledgments}

This work was supported by PARTEX OIL AND GAS. The authors acknowledge the financial support from Fundação para a Ciência e a Tecnologia for the Pest-C/CTM/LA0011/2011 and doctoral research grant of Jorge F.B. Pereira SFRH/ BD/60228/2009.

\section{References}

1. J.D. Desai and I.M.Banat, "Microbial production of surfactants and their commercial potential", Microbiol. Mol. Biol. R. 61, 47-64 (1997).

2. M. Nitschke, S.G.V.A.O. Costa and J. Contiero, "Rhamnolipid surfactants: An update on the general aspects of these remarkable biomolecules", Biotechnol. Progr. 21, 1593-600 (2005). doi: $10.1021 /$ $\underline{\text { bp050239p }}$

3. S. Mukherjee, P. Das and R. Sen, "Towards commercial production of microbial surfactants", Trends Biotechnol. 24, 509-15 (2006). doi: 10.1016/j.tibtech.2006.09.005

4. J.D. Van Hamme, A. Singh and O.P. Ward, "Surfactants in microbiology and biotechnology: Part 1. Physiological aspects", Biotechnol. Adv. 24, 604-20 (2006). doi: 10.1016/j.biotechadv.2006.08.001

5. A. Singh, J.D. Van Hamme and O.P. Ward, “Surfactants in microbiology and biotechnology: Part 2. Application aspects", Biotechnol. Adv. 25, 99-121 (2007). doi: 10.1016/j.biotechadv.2006.10.004

6. Z.A. Raza, Z.M. Khalid and I.M. Banat, "Characterization of rhamnolipids produced by a Pseudomonas aeruginosa mutant strain grown on waste oils", J. Environ. Sci. Heal. A 44, 1367-73 (2009).

7. C.N. Mulligan, "Environmental applications for biosurfactants", Environ. Pollut. 133, 183-98 (2005). doi: 10.1016/j.envpol.2004.06.009

8. G. Soberon-Chavez, F. Lepine and E. Deziel, "Production of rhamnolipids by Pseudomonas aeruginosa", Appl Microbiol Biot. 68, 718-25 (2005). doi: 10.1007/s00253005-0150-3

9. A. Perfumo, I.M. Banat, F. Canganella and R. Marchant, "Rhamnolipid production by a novel thermophilic hydrocarbon-degrading Pseudomonas aeruginosa AP021". Appl. Microbiol. Biot. 72, 132-8 (2006). doi: $10.1007 /$ s00253-005-0234-0

10. S.A. Monteiro, G.L. Sassaki, L.M. de Souza, J.A. Meira, J.M. de Araujo, D.A. Mitchell, L.P. Ramos and N. Krieger, "Molecular and structural characterization of the biosurfactant produced by Pseudomonas aeruginosa DAUPE 614", Chem. Phys. Lipids 147, 1-13 (2007). doi: 10.1016/i. chemphyslip.2007.02.001 
11. A.M. Abdel-Mawgoud, F. Lepine and E. Deziel, "Rhamnolipids: diversity of structures, microbial origins and roles", Appl. Microbiol. Biot. 86, 1323-36 (2010). doi: 10.1007/s00253-010-2498-2

12. S. Milne, P. Ivanova, J. Forrester and H.A. Brown, "Lipidomics: An analysis of cellular lipids by ESI-MS", Methods 39, 92-103 (2006). doi: 10.1016/j. ymeth.2006.05.014

13. J. Zaia, "Mass spectrometry of oligosaccharides", Mass Spectrom. Rev. 23, 161-227 (2004). doi: 10.1002/ mas.10073

14. M. Heyd, A. Kohnert, T.H. Tan, M. Nusser, F. Kirschhofer, G. Brenner-Weiss, M. Franzreb and S. Berensmeier, "Development and trends of biosurfactant analysis and purification using rhamnolipids as an example", Anal. Bioanal. Chem. 391, 1579-90 (2008). doi: 10.1007/s00216007-1828-4

15. H. Yin, J. Qiang, Y. Jia, J.S. Ye, H. Peng, H. Qin, N. Zhang and B. He, "Characteristics of biosurfactant produced by Pseudomonas aeruginosa S6 isolated from oil-containing wastewater", Process Biochem. 44, 302-8 (2009). doi: 10.1016/j.procbio.2008.11.003

16. J.R. de Sousa, J.A.D. Correia, J.G.L. de Almeida, S. Rodrigues, O.D.L. Pessoa, V.M.M. Melo and L.R.B. Gonçalves, "Evaluation of a co-product of biodiesel production as carbon source in the production of bio surfactant by $P$. aeruginosa MSIC02", Process Biochem. 46, 1831-9 (2011). doi: 10.1016/j. procbio.2011.06.016

17. Y.H. Wei, C.L. Chou and J.S. Chang, "Rhamnolipid production by indigenous Pseudomonas aeruginosa J4 originating from petrochemical wastewater", Biochem. Eng. J. 27, 146-54 (2005). doi: 10.1016/j.bej.2005.08.028

18. M.G. Rikalovic, G. Gojgic-Cvijovic, M.M. Vrvic and I. Karadzic, "Production and characterization of rhamnolipids from Pseudomonas aeruginosa san-ai. J. Serb. Chem. Soc. 77, 27-42 (2012). doi: 10.2298/JSC110211156R
19. N.P.J. Price, K.J. Ray, K. Vermillion and T.M. Kuo, "MALDI-TOF mass spectrometry of naturally occurring mixtures of monorhamnolipids and dirhamnolipids", Carbohyd. Res. 344, 204-9 (2009). doi: 10.1016/j. carres.2008.10.013

20. E.J. Gudiña, J.F.B. Pereira, L.R. Rodrigues, J.A.P. Coutinho and J.A. Teixeira, "Isolation and study of microorganisms from oil samples for application in microbial enhanced oil recovery", Int. Biodeter. Biodegrad. 68, 56-64 (2012). doi: 10.1016/j.ibiod.2012.01.001

21. J. Folch, M. Lees and G.H.S. Stanley, "A simple method for the isolation and purification of total lipides from animal tissues", J. Biol. Chem. 226, 497-509 (1957).

22. J.C. Mata-Sandoval, J. Karns and A. Torrents, "Highperformance liquid chromatography method for the characterization of rhamnolipid mixtures produced by Pseudomonas aeruginosa UG2 on corn oil", J. Chromatogr. A 864, 211-20 (1999). doi: 10.1016/S0021-9673(99)00979-6

23. E. Haba, A. Abalos, O. Jauregui, M.J. Espuny and A. Manresa, "Use of liquid chromatography-mass spectroscopy for studying the composition and properties of rhamnolipids produced by different strains of Pseudomonas aeruginosa", J. Surfactants Deterg. 6, 15561 (2003). doi: 10.1007/s11743-003-0260-7

24. E. Haba, A. Pinazo, O. Jauregui, M.J. Espuny, M.R. Infante and A. Manresa, "Physicochemical characterization and antimicrobial properties of rhamnolipids produced by Pseudomonas aeruginosa 47T2 NCBIM 40044", Biotechnol. Bioeng. 81, 316-22 (2003). doi: $10.1002 /$ bit.10474

25. A.M. Pajarron, C.G. Dekoster, W. Heerma, M. Schmidt and J. Haverkamp, "Structure identification of natural rhamnolipid mixtures by fast-atom-bombardment tandem mass-spectrometry", Glycoconjugate J. 10, 219-26 (1993). doi: http://dx.doi.org/10.1007/BF00702203 